\title{
Wide Diameters of Cartesian Product Graphs and Digraphs*
}

JUN-MING XU

xujm@ustc.edu.cn

Department of Mathematics, University of Science and Technology of China, Hefei, Anhui, 230026, China

Received September 15, 2000; Revised January 23, 2003; Accepted January 24, 2003

\begin{abstract}
In graph theory and study of fault tolerance and transmission delay of networks, connectivity and diameter of a graph are two very important parameters and have been deeply studied by many authors. Wide diameter combining connectivity with diameter is a more important parameter to measure fault tolerance and efficiency of parallel processing computer networks and has received much attention in the recent years. Diameter with width $k$ of a graph $G$ is defined as the minimum integer $d$ for which between any two distinct vertices in $G$ there exist at least $k$ internally disjoint paths of length at most $d$. In the present paper, the tight upper bounds of wide diameter of the Cartesian product graphs are obtained. Some known results can be deduced or improved from ours.
\end{abstract}

Keywords: connectivity, diameter, wide diameter, cartesian product, networks, fault tolerance

AMS Subject Classification: 05C12, 05C40, 68M10

\section{Introduction}

In this paper, the letter $G$ always stands for a finite, simple and connected graph or digraph from the context with the vertex-set $V=V(G)$ and the edge (or arc)-set $E=E(G)$. The term graph means a undirected graph, digraph means a directed graph, and connected for digraphs means strongly connected. We follow Bondy and Murty (1976) for terminology and notation not defined and explained here.

Consider $G$ as a model of a computer interconnection network with each vertex representing a processor and each edge or arc representing a two- or one-way communication link. Fault tolerance and efficiency are important criteria in design of interconnection networks. Fault tolerance and transmission delay of the networks are often measured by, respectively, connectivity and diameter of the corresponding graph or digraph, which have been deeply studied by many authors. The advent of VLSI technology and fiber optics material science has enabled us to make very large scale parallel processing computer systems, and fast and complicated communication networks. To consider fault tolerance and efficiency, all these systems demand the existence of a large number of internally disjoint paths connecting any two vertices, each of which is of short length. In the circumstances, any exclusive consideration of connectivity or diameter is not comprehensive. This issue motivates us to consider the following concepts by combining connectivity with diameter rather naturally.

*The work was supported by ANSF (No. 01046102) and NNSF of China (No.10271114). 
Let $G$ be a connected graph or digraph, and $x, y$ be two distinct vertices of $G$. The distance with width $k$ from $x$ to $y, k$-distance for short and denoted by $d_{k}(G ; x, y)$, is the minimum integer $d$ for which there are at least $k$ internally disjoint $(x, y)$-paths of length at most $d$ in $G$. The diameter with width $k$ of $G, k$-diameter for short and denoted by $d_{k}(G)$, is defined as the maximum $k$-distance $d_{k}(G ; x, y)$ over all ordered pairs $(x, y)$ of vertices of $G$.

The concept of wide diameter is first proposed by Hsu (1994), Hsu and Lyuu (1991), Hsu and Luczak (1994), Flandrin and Li (1994), independently. From the definition of wide diameter, it is clear that $d_{1}(G)$ is the diameter $d(G)$ and

$$
d_{k}(G) \geq d_{k-1}(G) \geq \cdots \geq d_{2}(G) \geq d_{1}(G)=d(G)
$$

This implies that wide diameter is a generalization of diameter. If $G$ is $k$-connected, i.e., the connectivity $\kappa(G) \geq k$, then $d_{k}(G)$ certainly exists by the well-known Menger's theorem (see Bondy and Murty, 1976, Theorem 11.6 and Corollary 11.7). The maximum value of $k$ that $d_{k}(G)$ is well defined is the connectivity $\kappa(G)$. Thus the concept of wide diameter is a combination of connectivity and diameter. It follows that wide diameter is not only an important parameter to measure fault tolerance and efficiency of parallel processing computer networks, but also an attractive research topic in graph theory. It has received much attention in recent years (Cao et al., 1999; Du et al., 1993; Duh et al., 1996; Flandrin and Li, 1994; Hayes and Mudge, 1989; Hsu, 1994; Hsu and Lyuu, 1991; Hsu and Luczak, 1994; Ishigami, 1996; Jwo and Tuan, 1999; Kirshnamoorthy and Krinamurthy, 1987; Li et al., 1996; Liaw and Chang, 1999a, 1999b; Saad and Schultz, 1988).

As an operation of graphs, the Cartesian product is an important method in designing very large scale networks from small ones. Hsu (1994) considered wide diameter of the Cartesian product $G_{1} \times G_{2}$ and proved that $d_{k_{1}+k_{2}}\left(G_{1} \times G_{2}\right) \leq d_{k_{1}}\left(G_{1}\right)+d_{k_{2}}\left(G_{2}\right)$ if $G_{i}$ is a $k_{i}(\geq 1)$ connected graph with order at least three for each $i=1,2$. In this paper, we will improve this result by considering wide diameter of the Cartesian product $G_{1} \times G_{2} \times \cdots \times G_{n}$ of $n$ graphs (resp. digraphs) $G_{1}, G_{2}, \ldots, G_{n}$.

To state our results in this paper, we need introduce a concept. A $k$-connected (di)graph $G$ is said to be good if for any vertex $x$ in $G$ and any $k$ (out-)neighbors $v_{1}, v_{2}, \ldots, v_{k}$ of $x$ there are at least $k$ internally disjoint $\left(v_{i}, x\right)$-paths of length at most $d(G)$. Clearly, any $k$-connected graph is good.

Now, we can state our main results in this paper as follows.

Theorem A. For $i=1,2, \ldots, n$, let $G_{i}$ be a good and $k_{i}(\geq 1)$ connected digraph. If $G=G_{1} \times G_{2} \times \cdots \times G_{n}$ and $k=k_{1}+k_{2}+\cdots+k_{n}$, then

$$
\begin{aligned}
d_{k}(G) \leq & \max \left\{d\left(G_{1}\right)+\cdots+d\left(G_{n}\right)+1, d\left(G_{1}\right)+\cdots+d\left(G_{i-1}\right)\right. \\
& \left.+d_{k_{i}}\left(G_{i}\right)+d\left(G_{i+1}\right)+\cdots+d\left(G_{n}\right): 1 \leq i \leq n\right\} .
\end{aligned}
$$

Theorem B. For wide diameter of the Cartesian products of graphs, we have the following three conclusions. 
(i) Let $G_{i}$ be a graph with connectivity $k_{i}(\geq 1)$ and order at least three for $i=1,2, \ldots, n$. If $G=G_{1} \times G_{2} \times \cdots \times G_{n}$ and $k=k_{1}+k_{2}+\cdots+k_{n}$, then

$$
\begin{aligned}
d_{k}(G) \leq & \max \left\{d\left(G_{1}\right)+\cdots+d\left(G_{i-1}\right)+d_{k_{i}}\left(G_{i}\right)\right. \\
& \left.+d\left(G_{i+1}\right)+\cdots+d\left(G_{n}\right): 1 \leq i \leq n\right\}
\end{aligned}
$$

(ii) Let $G$ be a graph with connectivity $k(\geq 2)$. Then $d_{1+k}\left(K_{2} \times G\right) \leq d(G)+2$ if $d_{k}(G)=$ $d(G)+1$.

(iii) Let $G_{i}$ be a graph with connectivity $k_{i}(\geq 1)$ for $i=1,2$. Then $d_{k_{1}+k_{2}}\left(G_{1} \times G_{2}\right) \geq$ $d\left(G_{i}\right)+2$ if $G_{i}$ is $k_{i}$-regular for $i=1$ or 2 .

The rest of this paper is organized as follows. In Section 2, the definition and some fundamental properties of the Cartesian products are given. The proofs of Theorems A and $\mathrm{B}$ are given in Section 3. Other results, corollaries and some remarks are given in Section 4. In Conclusions, we propose a conjecture.

\section{Fundamental properties of cartesian products}

The Cartesian product of $n$ digraphs $G_{1}, G_{2}, \ldots, G_{n}$ is the digraph $G$, denoted by $G_{1} \times$ $G_{2} \times \cdots \times G_{n}$, with the vertex-set $V(G)=V\left(G_{1}\right) \times V\left(G_{2}\right) \times \cdots \times V\left(G_{n}\right)$, and an arc from a vertex $x=x_{1} x_{2} \cdots x_{n}$ to another vertex $y=y_{1} y_{2} \cdots y_{n}\left(x_{j}, y_{j} \in V\left(G_{j}\right), j=1,2, \ldots, n\right)$ if and only if they differ in exactly one coordinate, and for this coordinate, say $j t h$, there is an arc from the vertex $x_{j}$ to the vertex $y_{j}$ in $G_{j}$.

Similarly, we can define the Cartesian product of $n$ graphs.

As an operation of graph theory, the Cartesian product has been widely used in designing large scale computer systems and interconnection networks (see Bermond et al., 1986). Thus it has been deeply investigated from many different perspectives. These studies have led to the discovery of many properties (Xu, 1998).

First, we point out that although graphs and digraphs are essentially different objects, a graph can in the circumstances be thought of as a digraph in which there are two arcs, one in each direction, corresponding to each edge. In view of this fact, a graph can be thought of as a special digraph. As a result, any result for digraphs has an analogy for graphs as well.

Secondly, we observe that if we identify isomorphic (di)graphs, the operations of the Cartesian products satisfy associative and commutative laws clearly. We have in the literature seen it is such a simple observation that can make us greatly simplify proofs of some results concerning the Cartesian products.

Thirdly, we note that if $P=\left(x_{1}, v_{1}, v_{2}, \ldots, v_{m}, y_{1}\right)$ is an $\left(x_{1}, y_{1}\right)$-path in $G_{1}$, then for any $b \in V\left(G_{2}\right),\left(x_{1} b, v_{1} b, v_{2} b, \ldots, v_{m} b, y_{1} b\right)$, denoted by $P b$, is an $\left(x_{1} b, y_{1} b\right)$-path from the vertex $x_{1} b$ to the vertex $y_{1} b$ in $G_{1} \times G_{2}$. Similarly, if $W=\left(x_{2}, u_{1}, u_{2}, \ldots, u_{l}, y_{2}\right)$ is an $\left(x_{2}, y_{2}\right)$-path in $G_{2}$, then for any $a \in V\left(G_{1}\right),\left(a x_{2}, a u_{1}, a u_{2}, \ldots, a u_{l}, a y_{2}\right)$, denoted by $a W$, is an $\left(a x_{2}, a y_{2}\right)$-path from the vertex $a x_{2}$ to the vertex $a y_{2}$ in $G_{1} \times G_{2}$. Let $x=x_{1} x_{2}$ and $y=y_{1} y_{2}$. If $x$ and $y$ are two distinct vertices in $G_{1} \times G_{2}$, then $P x_{2} \cup y_{1} W$ is an $(x, y)$-path 
from $x$ to $y$ in $G_{1} \times G_{2}$. Such a path will, in this paper, be expressed as

$$
x=x_{1} x_{2} \quad \stackrel{P x_{2}}{\longrightarrow} \quad y_{1} x_{2} \quad \stackrel{y_{1} W}{\longrightarrow} \quad y_{1} y_{2}=y .
$$

Lastly, we list two fundamental facts used in this paper about the Cartesian products, which are well-known and can be found in the literature (see, for example (Xu, 1998)).

Fact 1. $G_{1} \times G_{2} \times \cdots \times G_{n}$ is $k$-regular if $G_{i}$ is $k_{i}$-regular, and is $k$-connected if $G_{i}$ is $k_{i}(\geq 1)$-connected, where $k=k_{1}+k_{2}+\cdots+k_{n}$.

Fact 2. The diameter of $G_{1} \times G_{2} \times \cdots \times G_{n}$ is equal to $d\left(G_{1}\right)+d\left(G_{2}\right)+\cdots+d\left(G_{n}\right)$.

\section{Proofs of Theorem A and Theorem B}

Proof of Theorem A: $\quad$ Since $G$ is $k$-connected from Fact 1, $k$-diameter of $G d_{k}(G)$ is well defined. We will complete the proof by induction on $n \geq 2$. For $n=2$, we need only construct $k_{1}+k_{2}$ internally disjoint $(x, y)$-paths in $G_{1} \times G_{2}$ such that each of them is of length at most

$$
\max \left\{d_{k_{1}}\left(G_{1}\right)+d\left(G_{2}\right), d\left(G_{1}\right)+d_{k_{2}}\left(G_{2}\right), d\left(G_{1}\right)+d\left(G_{2}\right)+1\right\}
$$

for any two distinct vertices $x$ and $y$ in $G_{1} \times G_{2}$.

Let $x=x_{1} x_{2}$ and $y=y_{1} y_{2}$, where $x_{1}, y_{1} \in V\left(G_{1}\right)$ and $x_{2}, y_{2} \in V\left(G_{2}\right)$. Let $a_{i}=\left(x_{1}, v_{i}\right)$ be the out-arc from $x_{1}$ in $G_{1}$ for each $i=1,2, \ldots, d_{G_{1}}^{+}\left(x_{1}\right)$, and $b_{j}=\left(x_{2}, u_{j}\right)$ the out-arc from $x_{2}$ in $G_{2}$ for each $j=1,2, \ldots, d_{G_{2}}^{+}\left(x_{2}\right)$.

If $x_{1} \neq y_{1}$, then there exist a shortest $\left(x_{1}, y_{1}\right)$-path $P$ and $k_{1}$ internally disjoint $\left(x_{1}, y_{1}\right)$ paths $P_{1}, P_{2}, \ldots, P_{k_{1}}$ in $G_{1}$ such that $\varepsilon\left(P_{i}\right) \leq d_{k_{1}}\left(G_{1}\right)$ for each $i=1,2, \ldots, k_{1}$. We can, without loss of generality, suppose that $P_{i}$ has the first arc $a_{i}$ for each $i=1,2, \ldots, k_{1}$, and $P$ contains none of the arcs $a_{2}, \ldots, a_{k_{1}}$. Thus $\varepsilon\left(P_{i}\right) \geq 2$ for $i=2,3, \ldots, k_{1}$. Then $v_{i}$ cuts $P_{i}$ into two subpaths, $a_{i}$ and $P_{i}^{\prime}$, where $P_{i}^{\prime}$ is the $\left(v_{i}, y_{1}\right)$-section of $P_{i}$. And so the $\left(x_{1}, y_{1}\right)$-path $P_{i}$ can be expressed as

$$
P_{i}=x_{1} \stackrel{a_{i}}{\longrightarrow} v_{i} \stackrel{P_{i}^{\prime}}{\longrightarrow} y_{1}, \quad i=2,3, \ldots, k_{1}
$$

Similarly, if $x_{2} \neq y_{2}$, there are a shortest $\left(x_{2}, y_{2}\right)$-path $W$ and $k_{2}$ internally disjoint $\left(x_{2}, y_{2}\right)$-paths $W_{1}, W_{2}, \ldots, W_{k_{2}}$ in $G_{2}$ such that each of them is of length $\varepsilon\left(W_{j}\right) \leq d_{k_{2}}\left(G_{2}\right)$. Let, without loss of generality, $b_{j}=\left(x_{2}, u_{j}\right)$ be the first arc in $W_{j}$ for $j=1,2,3, \ldots, k_{2}$, and suppose that $W$ contains none of the $\operatorname{arcs} b_{2}, \ldots, b_{k_{2}}$. Then $u_{j}$ is an internal vertex in $W_{j}$ and

$$
W_{j}=x_{2} \stackrel{b_{j}}{\longrightarrow} u_{j} \stackrel{W_{j}^{\prime}}{\longrightarrow} y_{2}, \quad j=2,3, \ldots, k_{2},
$$

where $W_{j}^{\prime}$ is the $\left(u_{j}, y_{2}\right)$-section of $W_{j}$ for each $j=2,3, \ldots, k_{2}$. 
Since $G_{1}$ is a good digraph, for the subset $\left\{v_{1}, v_{2}, \ldots, v_{k_{1}}\right\}$ of vertices in $G_{1}$, there must exist $k_{1}$ internally disjoint $\left(v_{i}, x_{1}\right)$-paths $T_{1}, T_{2}, \ldots, T_{k_{1}}$ in $G_{1}$ such that each of them is of length at most $d\left(G_{1}\right)$ for $i=1,2, \ldots, k_{1}$. Similarly, there are $k_{2}$ internally disjoint $\left(u_{j}, x_{2}\right)$-paths $U_{1}, U_{2}, \ldots, U_{k_{2}}$ in $G_{2}$ such that each of them is of length at most $d\left(G_{2}\right)$ for $j=1,2, \ldots, k_{2}$.

Using the above notation, we construct $k_{1}+k_{2}$ internally disjoint $\left(x_{1} x_{2}, y_{1} y_{2}\right)$-paths $R_{1}, R_{2}, \ldots, R_{k_{1}+k_{2}}$ in $G_{1} \times G_{2}$ as follows.

If $y_{1} \neq x_{1}$ and $y_{2} \neq x_{2}$, then let

$$
\left\{\begin{array}{l}
R_{1}=x_{1} x_{2} \stackrel{P x_{2}}{\longrightarrow} y_{1} x_{2} \stackrel{y_{1} W_{1}}{\longrightarrow} y_{1} y_{2}, \\
R_{i}=x_{1} x_{2} \stackrel{a_{i}}{\longrightarrow} v_{i} x_{2} \stackrel{v_{i} W}{\longrightarrow} v_{i} y_{2} \stackrel{P_{i}^{\prime} y_{2}}{\longrightarrow} y_{1} y_{2}, \quad i=2, \ldots, k_{1}, \\
R_{k_{1}+1}=x_{1} x_{2} \stackrel{x_{1} W}{\longrightarrow} x_{1} y_{2} \stackrel{P_{1} y_{2}}{\longrightarrow} y_{1} y_{2}, \\
R_{k_{1}+j}=x_{1} x_{2} \stackrel{x_{1} b_{j}}{\longrightarrow} x_{1} u_{j} \stackrel{P u_{j}}{\longrightarrow} y_{1} u_{j} \stackrel{y_{1} W_{j}^{\prime}}{\longrightarrow} y_{1} y_{2}, \quad j=2, \ldots, k_{2} .
\end{array}\right.
$$

Each of which is of length

$$
\left\{\begin{array}{l}
\varepsilon\left(R_{1}\right) \leq d\left(G_{1}\right)+d_{k_{2}}\left(G_{2}\right), \\
\varepsilon\left(R_{i}\right) \leq d_{k_{1}}\left(G_{1}\right)+d\left(G_{2}\right), \quad i=2,3, \ldots, k_{1}, \\
\varepsilon\left(R_{k_{1}+1}\right) \leq d_{k_{1}}\left(G_{1}\right)+d\left(G_{2}\right), \\
\varepsilon\left(R_{k_{1}+j}\right) \leq d\left(G_{1}\right)+d_{k_{2}}\left(G_{2}\right), \quad j=2,3, \ldots, k_{2} .
\end{array}\right.
$$

If $y_{1}=x_{1}$ and $y_{2} \neq x_{2}$, then let

$$
\left\{\begin{array}{l}
R_{i}=x_{1} x_{2} \stackrel{a_{i} x_{2}}{\longrightarrow} v_{i} x_{2} \stackrel{v_{i} W}{\longrightarrow} v_{i} y_{2} \stackrel{T_{i} y_{2}}{\longrightarrow} x_{1} y_{2}=y_{1} y_{2}, \quad i=1,2, \ldots, k_{1}, \\
R_{k_{1}+j}=x_{1} x_{2} \stackrel{x_{1} W_{j}}{\longrightarrow} x_{1} y_{2}=y_{1} y_{2}, \quad j=1,2, \ldots, k_{2} .
\end{array}\right.
$$

Each of which is of length

$$
\left\{\begin{array}{l}
\varepsilon\left(R_{i}\right) \leq 1+d\left(G_{2}\right)+d\left(G_{1}\right), \quad i=1,2, \ldots, k_{1}, \\
\varepsilon\left(R_{k_{1}+j}\right) \leq d_{k_{2}}\left(G_{2}\right), \quad j=1,2, \ldots, k_{2}
\end{array}\right.
$$

If $y_{1} \neq x_{1}$ and $y_{2}=x_{2}$, then let

$$
\left\{\begin{array}{l}
R_{i}=x_{1} x_{2} \stackrel{P_{i} x_{2}}{\longrightarrow} y_{1} x_{2}=y_{1} y_{2}, \quad i=1,2, \ldots, k_{1}, \\
R_{k_{1}+j}=x_{1} x_{2} \stackrel{x_{1} b_{j}}{\longrightarrow} x_{1} u_{j} \stackrel{P u_{j}}{\longrightarrow} y_{1} u_{j} \stackrel{x_{1} U_{j}}{\longrightarrow} y_{1} x_{2}=y_{1} y_{2}, \quad j=1,2, \ldots, k_{2} .
\end{array}\right.
$$

Each of which is of length

$$
\left\{\begin{array}{l}
\varepsilon\left(R_{i}\right) \leq d_{k_{1}}\left(G_{1}\right), \quad i=1,2, \ldots, k_{1}, \\
\varepsilon\left(R_{k_{1}+j}\right) \leq 1+d\left(G_{1}\right)+d\left(G_{2}\right), \quad j=1,2, \ldots, k_{2}
\end{array}\right.
$$


It follows that the induction base holds.

Assume Theorem A holds for $n-1$ and consider $n(\geq 3)$. Let $H=G_{2} \times G_{3} \times \cdots \times G_{n}$, $G=G_{1} \times H$ and $h=k_{2}+k_{3}+\cdots+k_{n}$. By the associativity of Cartesian product, induction base and induction hypothesis, we have that

$$
\begin{aligned}
d_{k}(G) \leq & \max \left\{d_{k_{1}}\left(G_{1}\right)+d(H), d\left(G_{1}\right)+d_{h}(H), d\left(G_{1}\right)+d(H)+1\right\} \\
\leq & \max \left\{d_{k_{1}}\left(G_{1}\right)+d\left(G_{2}\right)+\cdots+d\left(G_{n}\right), d\left(G_{1}\right)+\max \left\{d\left(G_{2}\right)+\cdots+d\left(G_{n}\right)+1,\right.\right. \\
& \left.d\left(G_{2}\right)+\cdots+d\left(G_{i-1}\right)+d_{k_{i}}\left(G_{i}\right)+d\left(G_{i+1}\right)+\cdots+d\left(G_{n}\right): 2 \leq i \leq n\right\}, \\
& \left.d\left(G_{1}\right)+d\left(G_{2}\right)+\cdots+d\left(G_{i}\right)+\cdots+d\left(G_{n}\right)+1\right\} \\
= & \max \left\{d\left(G_{1}\right)+\cdots+d\left(G_{i}\right)+\cdots+d\left(G_{n}\right)+1, d\left(G_{1}\right)+\cdots+d\left(G_{i-1}\right)\right. \\
& \left.+d_{k_{i}}\left(G_{i}\right)+d\left(G_{i+1}\right)+\cdots+d\left(G_{n}\right): 1 \leq i \leq n\right\} .
\end{aligned}
$$

The proof of Theorem A is completed.

Proof of Theorem B: First, we prove the first conclusion. Noting that any $m$-connected graph is good, we reach this aim by using the same statements as ones in the proof of Theorem A. We need only prove that

$$
d_{k_{1}+k_{2}}\left(G_{1} \times G_{2} ; x, y\right) \leq \max \left\{d_{k_{1}}\left(G_{1}\right)+d\left(G_{2}\right), d\left(G_{1}\right)+d_{k_{2}}\left(G_{2}\right)\right\}
$$

To this aim, we choose $k_{1}+k_{2}$ internally disjoint $(x, y)$-paths as the same as ones defined in (1), or (3), or (5). But in the circumstances, we replace $T_{i}$ in (3) by an edge $a_{i}^{\prime}=v_{i} x_{1}$ for each $i=1,2, \ldots, k_{1}$, and replace $U_{j}$ in (5) by an edge $b_{j}^{\prime}=u_{j} x_{2}$ for each $j=1,2, \ldots, k_{2}$. Thus, from the inequalities (2), (4) and (6), we need only consider the length of the paths $R_{1}, R_{2}, \ldots, R_{k_{1}}$ defined in (3) and $R_{k_{1}+1}, R_{k_{1}+2}, \ldots, R_{k_{1}+k_{2}}$ defined in (5), that is the length of the following paths

$$
\left\{\begin{array}{l}
R_{i}=x_{1} x_{2} \stackrel{a_{i} x_{2}}{\longrightarrow} v_{i} x_{2} \stackrel{v_{i} W}{\longrightarrow} v_{i} y_{2} \stackrel{a_{i}^{\prime} y_{2}}{\longrightarrow} x_{1} y_{2}=y_{1} y_{2}, i=1,2, \ldots, k_{1} \\
R_{k_{1}+j}=x_{1} x_{2} \stackrel{x_{1} b_{j}}{\longrightarrow} x_{1} u_{j} \stackrel{P u_{j}}{\longrightarrow} y_{1} u_{j} \stackrel{x_{1} b_{j}^{\prime}}{\longrightarrow} y_{1} x_{2}=y_{1} y_{2}, j=1,2, \ldots, k_{2} .
\end{array}\right.
$$

Clearly, these paths are of the lengths

$$
\left\{\begin{array}{l}
\varepsilon\left(R_{i}\right) \leq 2+d\left(G_{2}\right), i=1,2, \ldots, k_{1} \\
\varepsilon\left(R_{k_{1}+j}\right) \leq 2+d\left(G_{1}\right), j=1,2, \ldots k_{2}
\end{array}\right.
$$

It follows that, in order to prove (7), we need only show that

$$
\max \left\{d\left(G_{1}\right), d\left(G_{2}\right)\right\}+2 \leq \max \left\{d_{k_{1}}\left(G_{1}\right)+d\left(G_{2}\right), d\left(G_{1}\right)+d_{k_{2}}\left(G_{2}\right)\right\}
$$


In fact, note that either $d(G) \geq 2$ if $k=1$ or $d_{k}(G) \geq 2$ if $k \geq 2$ for any graph $G$ with connectivity $k(\geq 1)$ and order at least three, which implies that the inequality (9) holds clearly. Thus the first conclusion in Theorem B follows.

We now prove the second conclusion. Note that under our assumption, $d\left(K_{2}\right)+d_{k}(G)=$ $d(G)+2$. Also note that, in the above discussion, the assumption of order at least three is used in the proof of the inequality (9). But this is clear for $G_{1}=K_{2}$ and $G_{2}=G$ since $\max \left\{d\left(K_{2}\right), d(G)\right\}+2=d(G)+2=1+d_{k}(G)=\max \left\{d\left(K_{2}\right)+d(G), d\left(K_{2}\right)+d_{k}(G)\right\}$.

Next, we show the third conclusion. In fact, if $G_{1}$ is $k_{1}$-regular, then let $x_{1}, y_{1} \in V\left(G_{1}\right)$ such that distance between $x_{1}$ and $y_{1}$ in $G_{1}$ is equal to $d\left(G_{1}\right)$. For any $x_{2} \in V\left(G_{2}\right)$, let $x=x_{1} x_{2}$ and $y=y_{1} x_{2}$, then $x$ and $y$ are two distinct vertices in $G_{1} \times G_{2}$ since $x_{1} \neq x_{2}$. Since $G_{1}$ is $k_{1}$-regular, of any $k_{1}+k_{2}$ paths between $x$ and $y$ in $G_{1} \times G_{2}$, at least one is constructed in the same way as (8), its length is $d\left(G_{1}\right)+2$. It follows that $d_{k_{1}+k_{2}}\left(G_{1} \times G_{2}\right) \geq d\left(G_{1}\right)+2$.

If $G_{2}$ is $k_{2}$-regular, then let $x_{2}, y_{2} \in V\left(G_{2}\right)$ such that distance between $x_{2}$ and $y_{2}$ in $G_{2}$ is equal to $d\left(G_{2}\right)$. Let $x=x_{1} x_{2}$ and $y=x_{1} y_{2}$. Then, similarly, we have $d_{k_{1}+k_{2}}\left(G_{1} \times G_{2}\right) \geq$ $d\left(G_{2}\right)+2$.

The proof of Theorem B is completed.

\section{Other results, corollaries and remarks}

It is possible for some graphs $G$ that wide diameter $d_{k}(G)$ is equal to its diameter $d(G)$, where $k(\geq 2)$ is connectivity of $G$. For instance, the $n(\geq 2)$-dimensional binary undirected de Bruijn graph $B(2, n)$ has connectivity 2 and diameter $n$. Li et al. (1996) showed that wide diameter $d_{2}(B(2, n))=n=d(B(2, n))$. However, Hsu and Luczak (1994) proved that $d_{k}(G) \geq d(G)+1$ for any $k$-regular $k$-connected graph $G$ if $k \geq 2$. We generalize this result to digraphs.

Theorem C. Let $G$ be a k-regular $k$-connected digraph. Then $d_{k}(G) \geq d(G)+1$ if $k \geq 2$.

Proof: Let $x$ and $y$ be two vertices of the digraph $G$ such that distance from $x$ to $y$ is equal to $d(G)$, and let $z$ be an out-neighbor of $y$. Consider any $k(\geq 2)$ internally disjoint $(x, z)$ paths in $G$. Of which at least one must contain $y$ and is of length at least $d(G)+1$. So $d_{k}(G) \geq d_{k}(G ; x, y) \geq d(G)+1$.

A $k$-regular $k$-connected graph or digraph $G$ satisfying $d_{k}(G)=d(G)+1$ is said to be $k$-optimal, where $k(\geq 2)$ is connectivity of $G$.

Corollary 1. $K_{2} \times G$ is $(k+1)$-optimal if the graph $G$ is k-optimal.

Proof: Note that $d_{k}(G)=d(G)+1, G$ is $k$-regular since $G$ is $k$-optimal, and $d\left(K_{2} \times G\right)=$ $1+d(G)$ by Fact 2 . By the second conclusion of Theorem B, we have that $d_{k+1}\left(K_{2} \times G\right) \leq$ $d(G)+2=1+d\left(K_{2} \times G\right)$.

On the other hand, since $G$ is $k$-regular $k$-connected, $K_{2} \times G$ is $(k+1)$-regular and $(k+1)$-connected by Fact 1 . It follows from Theorem C that $d_{k+1}\left(K_{2} \times G\right) \geq 1+d\left(K_{2} \times G\right)$. Hence $K_{2} \times G$ is $(k+1)$-optimal. 
Note: The inequality $d_{k_{1}+k_{2}}\left(K_{2} \times G\right) \geq d(G)+2$ is straightforward from the third conclusion of Theorem B.

Corollary 2. Let $G_{i}$ be a $k_{i}(\geq 2)$-optimal graph for each $i=1,2, \ldots, n(\geq 2)$, and let $k=k_{1}+k_{2}+\cdots+k_{n}$. Then $G=G_{1} \times G_{2} \times \cdots \times G_{n}$ is $k$-optimal.

Proof: Since for each $i=1,2, \ldots, n, G_{i}$ is $k_{i}$-optimal, $G_{i}$ is $k_{i}$-regular and $k_{i}$-connected. Thus $G$ is $k$-regular $k$-connected by Fact 1 . It follows that $d_{k}(G) \geq d(G)+1$ by Theorem C.

On the other hand, $d_{k_{i}}\left(G_{i}\right)=d\left(G_{i}\right)+1$ since $G_{i}$ is $k_{i}$-optimal for each $i=1,2, \ldots, n$. By Fact 2 the diameter $d(G)=d\left(G_{1}\right)+d\left(G_{2}\right)+\cdots+d\left(G_{n}\right)$. It follows from Theorem B that $d_{k}(G) \leq d\left(G_{1}\right)+d\left(G_{2}\right)+\cdots+d\left(G_{n}\right)+1=d(G)+1$. Thus $G$ is $k$-optimal.

Constructing $k$-optimal graphs or digraphs is one of main aims of network designers. A large number of such graphs and digraphs have been constructed in the literature. Some of them can be expressed as the Cartesian products, and some results can be deduced from ours directly.

The hypercube $Q_{k}$, a well-known topological structure of network, can be expressed as the Cartesian product $K_{2} \times K_{2} \times \cdots \times K_{2}$ of $k$ complete graphs $K_{2} . Q_{k}$ is $k$-regular $k$-connected, and has diameter $k$ by Fact 1 and Fact 2.

Corollary 3 (Hayes and Mudge, 1989; Kirshnamoorthy and Krinamurthy, 1987; Saad and Schultz, 1988). $\quad Q_{k}$ is $k$-optimal if $k \geq 2$.

Proof: We proceed by induction on $k \geq 2$. Since $Q_{2}=K_{2} \times K_{2}=C_{4}$, it is clear that the cycle $C_{4}$ is 2-optimal. Suppose that $Q_{k-1}$ is $(k-1)$-optimal. Consider $Q_{k}, k \geq 3$. Since $Q_{k}=K_{2} \times Q_{k-1}$ and $Q_{k-1}$ is $(k-1)$-optimal, it is obtained immediately that $Q_{k}$ is $k$-optimal by Corollary 1 .

The generalized hypercube $G\left(m_{1}, m_{2}, \ldots, m_{n}\right)$, proposed by Bhuyan and Agrawal (1984), can be expressed as $K_{m_{1}} \times K_{m_{2}} \times \cdots \times K_{m_{n}}$, where $m_{i} \geq 3$ for any $i=1,2, \ldots, n$. $G\left(m_{1}, m_{2}, \ldots, m_{n}\right)$ is $k$-regular $k$-connected, and has diameter $n$ from Fact 1 and Fact 2, where $k=m_{1}+m_{2}+\cdots m_{n}-n$.

Corollary 4 (Duh et al., 1996). $\quad G\left(m_{1}, m_{2}, \ldots, m_{n}\right)$ is $k$-optimal, where $k=m_{1}+m_{2}+$ $\cdots m_{n}-n$.

Proof: This is a direct consequence of Corollary 2 since $K_{m_{i}}$ is $\left(m_{i}-1\right)$-optimal.

We use $\vec{C}_{d}$ to denote a directed cycle of length $d(\geq 3)$, which has diameter $d-1$. The generalization of hypercube for digraphs $\vec{C}\left(d_{1}, d_{2}, \ldots, d_{k}\right)$, proposed by Hsu and Lyuu (1991), can be expressed as $\vec{C}_{d_{1}} \times \vec{C}_{d_{2}} \times \cdots \times \vec{C}_{d_{k}}$. It is easy to see that $\vec{C}\left(d_{1}, d_{2}, \ldots, d_{k}\right)$ is a $k$-regular $k$-connected digraph, and has diameter $h=d_{1}+d_{2}+\cdots+d_{k}-k$ from Fact 1 and Fact 2.

Corollary 5 (Hsu and Lyuu, 1991). $\quad \vec{C}\left(d_{1}, d_{2}, \ldots, d_{k}\right)$ is $k$-optimal if $k \geq 2$. 
Proof: let $G=\vec{C}\left(d_{1}, d_{2}, \ldots, d_{k}\right)$. Noting that $\vec{C}_{d_{i}}$ is good, by Theorem A, we have

$$
d_{k}(G) \leq \sum_{i=1}^{k} d\left(C_{d_{i}}\right)+1=\sum_{i=1}^{k}\left(d_{i}-1\right)+1=h=d(G)+1 .
$$

On the other hand, from Theorem C we have $d_{k}(G) \geq d(G)+1$. It follows that $d_{k}(G)=$ $d(G)+1$, which implies that $G$ is $k$-optimal.

Similarly, we use $C\left(d_{1}, d_{2}, \ldots, d_{k}\right)$ to express the Cartesian product $C_{d_{1}} \times C_{d_{2}} \times \cdots \times C_{d_{k}}$, which is called the torodal mesh by Ishigami (1996). It is clear that $C\left(d_{1}, d_{2}, \ldots, d_{k}\right)$ is a $2 k$-regular $2 k$-connected graph from Fact 1 and Fact 2.

Corollary 6 (Ishigami, 1996). $d_{4}(C(3, n))=n$ if $n \geq 3$.

Proof: Note that $d\left(C_{3}\right)=1$ and $d_{2}\left(C_{n}\right)=n-1$. Let $x=x_{1} x_{2}$ and $y=y_{1} y_{2}$ be the two vertices in $C(3, n)$, where $x_{1} y_{1} \in E\left(C_{3}\right)$ and $x_{2} y_{2} \in E\left(C_{n}\right)$. The structure of any four internally disjoint $(x, y)$ paths in $C(3, n)$ must be similar to one in (2). Of these paths, at least one is of length at least $n$. This implies $d_{4}(C(3, n)) \geq n$.

On the other hand, by Theorem B, we have $d_{4}(C(3, n)) \leq n$ if $n \geq 3$.

Remark 1. Corollary 5 shows that the upper bound in Theorem A can be reached.

Remark 2. Corollary 6 shows that the upper bound in (i) of Theorem B can be reached. Corollary 1 shows that the upper bound in (ii) of Theorem B and the lower bound in (iii) of Theorem B can be reached.

Remark 3. The condition "order at least three" is necessary to in the first conclusion of Theorem B. A simple example is $C_{4}=K_{2} \times K_{2}$. A more complicated example is $G=K_{2} \times B(2, n)$, where $B(2, n)$ is the $n(\geq 2)$-dimensional binary undirected de Bruijn graph mentioned in the beginning of this section. $B(2, n)$ has the connectivity 2 and the diameter $n$. From the first conclusion of Theorem $\mathrm{B}$, we have that $d_{3}(G) \leq n+1$. But from the third conclusion of Theorem $\mathrm{B}$, we have that $d_{3}(G) \geq n+2$. This is a contradiction.

In addition, the condition " $G_{1}$ is $k_{1}$-regular or $G_{2}$ is $k_{2}$-regular" is indispensable to in the second conclusion of Theorem B.

Remark 4. Hsu (1994, Theorem 2.8) proved that $d_{k_{1}+k_{2}}\left(G_{1} \times G_{2}\right) \leq d_{k_{1}}\left(G_{1}\right)+d_{k_{2}}\left(G_{2}\right)$ if $G_{i}$ is a $k_{i}(\geq 1)$-connected graph with order at least three for each $i=1,2$. Obviously, this is a direct consequence of the first conclusion in Theorem B for $n=2$ since $d_{m}(H) \geq$ $d(H)$ for any $m$-connected graph $H$. However, Theorem B is stronger than Hsu's result if $d_{k_{1}}\left(G_{1}\right)>d\left(G_{1}\right)\left(\right.$ or $\left.d_{k_{2}}\left(G_{2}\right)>d\left(G_{2}\right)\right)$. This means that Theorem B improves Hsu's result.

Remark 5. A $k$-connected graph is called tight if for every pair of vertices $x$ and $y$ there are at least $k$ internally disjoint paths between $x$ and $y$ such that each of them is of length at most $d_{k}(G)$ and at least one is of length at most $d(G)$. Hsu (1994, Corollary 2.10) proved that 
$d_{k_{1}+k_{2}}\left(G_{1} \times G_{2}\right) \leq \max \left\{d_{k_{1}}\left(G_{1}\right)+d\left(G_{2}\right), d\left(G_{1}\right)+d_{k_{2}}\left(G_{2}\right)\right\}$ if $G_{i}$ is a tightly $k_{i}$-connected graph for $i=1,2$. The first conclusion of Theorem B shows that this result is true for any connected graphs. Thus, Theorem B improves Hsu's result.

\section{Conclusions}

In the present paper we have studied wide diameters of the Cartesian product graphs and digraphs, and obtained their tight upper bounds. In particular, for undirected graphs, these bounds only depend on diameters and wide diameters of the graphs as the product factors. We have deduced and improved some known results from ours. At the same time, we note that the first conclusion of Theorem B holds for any $k(\geq 1)$-connected graph with order at least three, while Theorem A holds under the assumption that as the product factors, all digraphs must be good. It is clear that every 1-connected digraph is good. We have not yet known if Theorem A holds for any $k(\geq 2)$-connected digraph. However we have the following conjecture.

Conjecture. $d_{k_{1}+k_{2}}\left(G_{1} \times G_{2}\right) \leq \max \left\{d_{k_{1}}\left(G_{1}\right)+d\left(G_{2}\right), d\left(G_{1}\right)+d_{k_{2}}\left(G_{2}\right)\right\}+1$ for any $k_{i}(\geq 2)-$ connected digraph $G_{i}, i=1,2$.

Note added in the revised version The original manuscript of this paper had been submitted to the journal for possible publication before the author had an attempt to write a book on topological structure and analysis of interconnection networks. When the manuscript is asked to revise the book $(\mathrm{Xu}, 2001)$ has been published, which, of course, contains the original result in the manuscript. One of two anonymous referees has noted this fact, and another has pointed out a flaw in the proof of Theorem A, that is Theorem 4.4.6 in $(\mathrm{Xu}, 2001)$. The author would like to publish this paper to correct the error, and to thank the two referees for their kind comments and valuable suggestions, which led to the revised version of the present paper.

\section{References}

J.-C. Bermond, C. Delorme, and J.-J. Quisquater, "Strategies for interconnection networks: Some methods from graph theory. J. Parallel Distri. Comput., vol. 3, pp. 433-449, 1986.

L.N. Bhuyan and D.P. Agrawal, "Generalized hypercube and hyperbus structures for a computer network," IEEE Trans. Comput., vol. 33, pp. 323-333, 1984.

J.A. Bondy and U.S.R. Murty, Graph Theory with Applications, Macmillan Press: London, 1976.

F. Cao, D.-Z. Du, D.F. Hsu, and S.H. Teng, "Fault tolerance properties of pyramid networks," IEEE Trans Comput., vol. 46, no. 1, pp. 88-93, 1999.

D.-Z. Du, Y.-D. Lyuu, and D.F. Hsu, "Line digraph iterations and connectivity analysis of de Bruijn and Kautz graphs," IEEE Trans. Computers, vol. 42, no. 5, pp. 612-616, 1993.

D.R. Duh, G.H. Chen, and D.F. Hsu, “Combinatorial properties of generalized hypercube graphs," Information Processing Letters, vol. 57, pp. 41-45, 1996.

E. Flandrin and H. Li, "Mengerian properties, Hamiltonicity and claw-free graphs," Networks, vol. 24, pp. 660-678, 1994.

J.P. Hayes and T.N. Mudge, "Hypercube supercomputers," Proc. IEEE, vol. 77, no. 12, pp. 1829-1841, 1989. 
D.H. Hsu, "On container width and length in graphs, groups, and networks," IEICE Trans, Fundam, vol. E 77A, pp. 668-680, 1994

D.H. Hsu and Y.D. Lyuu, "A graph-theoretical study of transmission delay and fault tolerance," in Proc. of 4th ISMM International Conference on Parallel and Distributed Computing and Systems, 1991, pp. 20-24.

D.F. Hsu and T. Luczak, "Note on the $k$-diameter of $k$-regular $k$-connected graphs," Discrete Math, vol. 132, pp. 291-296, 1994

Y. Ishigami, “The wide-diameter of the $n$-dimensional torodal mesh,” Networks, vol. 27, pp. 257-266, 1996.

J.S. Jwo and T.C. Tuan, "On container length and connectivity in unidirectional hypercubes," Networks, vol. 32 , pp. 307-317, 1999.

M.S. Kirshnamoorthy and B. Krinamurthy, "Fault diameter of interconnection networks," Comput. Math. Applic., vol. 13 , nos. 5/6, pp. 577-582, 1987.

Q. Li, D. Sotteau, and J.-M. Xu, "2-diameter of de Bruijn graphs,” Networks, vol. 28, no. 1, pp. 7-14, 1996.

S.C. Liaw and G.J. Chang, "Wide diameters of Butterfly networks," Taiwanese Journal of Mathematics, vol. 3 , no. 1, pp. 83-88, 1999a.

S.C. Liaw and G.J. Chang, "Generalized diameters and Rabin numbers of networks," J. Combinatorial Optimization, vol. 2, pp. 371-384, 1999 b.

Y. Saad and M.H. Schultz, "Topogical properties of hypercubes," IEEE Trans. Computers, vol. 37, no. 7, pp. 867-872, 1988.

J.-M. Xu, "Connectivity of Cartesian product digraphs and fault-tolerant routing of generalized hypercube," Appl. Math. -JCU, vol. 13B, no. 2, pp. 179-187, 1998.

J.-M. Xu, Topological Structure and Analysis of Interconnection Networks. Kluwer Academic Publishers: Dordrecht/Boston/London, 2001. 